\title{
CIENCIA DEL CONOCIMIENTO SENSIBLE: PRINCIPIOS RACIONALISTAS EN LA DOCTRINA ESTÉTICA DE ALEXANDER BAUMGARTEN*
}

\author{
Pablo Chiuminatto \\ Pontificia Universidad Católica de Chile \\ pchiuminatto@uc.cl
}

\begin{abstract}
Resumen / Abstract
A mediados del siglo XVIII, A. Baumgarten estableció una "ciencia del conocimiento sensible" o estética. Su propuesta ampliaba el legado de Leibniz y Wolff, profundizaba la noción de experiencia y asumía el esquema tradicional de la Idea (claridad y distinción) que Leibniz criticó a Descartes, pero considerando la acepción de ideas "simbólicas". No obstante su relevancia, la propuesta de Baumgarten no siempre es considerada para comprender la teoría del arte (o de lo 'bello', en general), que aparece como una vía menos relevante frente a la revolución kantiana de la estética asociada a la noción de juicio de gusto.
\end{abstract}

Palabras Clave: racionalismo, estética, poética, metaforología.

THE SCIENCE OF SENSITIVE KNOWLEDGE: RATIONALISTIC PRINCIPLES OF THE ESTHETICAL DOCTRINE OF ALEXANDER BAUMGARTEN

In the middle of the 18th century, A. Baumgarten established a science of sensitive knowledge also known as aesthetics. His proposal expanded the legacy from Leibniz and Wolff, it deepened the notion of experience and assumed the traditional model of the Idea (clarity and distinction) that Leibniz criticized to Descartes, while it still considered the meaning of "symbolic" ideas. Notwithstanding its relevance, Baumgarten's proposal is not always considered in the understanding of the theory of arts (or of 'the beautiful' in general), which appears to be a less relevant route when compared to the Kantian revolution of aesthetics associated to the notion of the judgment of taste.

KEY WORDS: rationalism; esthetics, poetics, metaphorology.

El presente artículo es parte de la investigación realizada gracias al proyecto Fondecyt $\mathrm{N}^{\circ} 11100135$, "Ciencia del conocimiento sensible: indagaciones sobre la influencia del racionalismo cartesiano en la estética de Alexander Baumgarten". 
$\overline{R M}$ J.-Y. Pranchère, en la presentación de la edición francesa de la AEsthetica de Alexander Baumgarten (1714-1762), asume que a pesar del interés que representa su obra, sin lugar a dudas, "no forma parte de los filósofos célebres" (7). Y aunque Baumgarten, en apariencia, por ejemplo en su Metafísica (1739), pareciera no hacer otra cosa que exponer in extenso los principios heredados de la filosofía de G. H. Leibniz (1646-1716), tal como los había entendido y posteriormente enseñado Chr. Wolff (1679-1754); su obra, escrita en latín, es considerada en general por la tradición como: “[...] un texto escolar sin originalidad real, en el que el único mérito sería representar, de manera ejemplar, una concepción anticuada de la filosofía -a saber, la idea prekantiana según la cual la metafísica puede y debe constituir una ciencia acabada y sistemática" (Pranchère 7).

De este modo, los textos de Baumgarten son descritos como una larga exposición interpretativa del "sistema Leibniz-Wolff". Es decir, son presentados como una consecuencia de un modelo anterior, extremadamente fuerte y brillante, que habría limitado la proyección autónoma de Baumgarten como filósofo. Sin embargo, su suerte - este destino epigonal- no es muy distinta de lo que ocurrió con el propio Wolff, quien también habría sucumbido bajo la fuerza del legado de Leibniz, sin conseguir entrar en el panteón que se levantó medio siglo después con la llegada, primero, de Immanuel Kant (1724-1824) y luego, especialmente, de G. W. F. Hegel (1770-1831) y su estética.

Las críticas que recibe Wolff sirven para entender el complejo panorama que determinó la recepción de un filósofo como Baumgarten porque, precisamente, sigue de cerca su doctrina general. Por una parte, se dice de Wolff que se limitaba a reproducir la filosofía de Leibniz; segundo, según expresas palabras de Kant en la Critica de la Razón Pura, Wolff es "el más grande de los filósofos dogmáticos", haciendo referencia al argumento ontológico de Dios (XXXVI); y -por último, tal como señala Gónzalez Ruiz en la selección de textos que hizo de la Metafísica alemana de Wolff al español-es fundamental considerar el hecho de que, entre las circunstancias que determinaron la omisión de la figura de Wolff, está el mismo Kant, quien se hizo cargo de destrozar el modelo wolffiano, al desmantelar, precisamente, aquel método filosófico (Metafisica 13).

Como decíamos antes, el caso de Baumgarten no es muy distinto del de su maestro. Reconocido históricamente como el fundador de la disciplina estética moderna, dicta en el año 1742 su primer curso sobre temas asociados a lo que hoy llamamos Estética, en la Universidad de Frankfurt. El acuñamiento del término, derivado del griego aisthesis, había sido anticipado por él, siguiendo a Aristóteles en De anima y los estudios de R. Descartes (1596-1650) en el Tratado de las pasiones (1649), en su disertación para acceder a la libre docencia, presentada en la Facultad de Filosofía de la Universidad de Halle, bajo el título Meditaciones filosóficas acerca del poema (Meditationes philosophica de nonnullis ad poema pertinentibus, 1735). Posteriormente, con la publicación de su Assthetica en dos volúmenes, de 1750 y 1758, respectivamente, instala definitivamente el término en el ámbito de la filosofía, entendido como "aquella ciencia que estudia el conocimiento sensible o lógica de las facultades cognoscitivas inferiores" (Baumgarten, Asthetica §1). 
En lo que respecta a la historia de la estética como disciplina, la obra de Baumgarten, debido a su temprano deceso, fue eclipsada -como decíamos anteriormente- por el halo desplegado en el contexto filosófico posterior y, en particular, por la potencia que tendrá la recepción y amplia influencia de la Tercera Crítica de Immanuel Kant (Kritik der Urteilskraft,1790). Paradójicamente, como es bien sabido, fueron concretamente las obras de Baumgarten las que sirvieron de base para los programas de los cursos que impartía el propio Kant. Borowsky, en Relato de la vida y el carácter de Immanuel Kant, menciona que el filósofo crítico, tal como era costumbre en la época, a la hora de enseñar, seguía a Ch. Wolff en Matemáticas, los manuales de G. F. Meier (1718-1777) para la Lógica, F. CH. Baumeister (1709-1788) primero y A. Baumgarten después para la Metafísica y, finalmente, J. A. Eberhard (1739-1809) para la Física (23).

Sin embargo, a pesar de este testimonio acerca de la importancia que tuvieron para Kant los escritos de Baumgarten, en la primera parte de la Crítica de la Razón Pura, en la sección referida a la Estética trascendental, Kant destina una única nota en la que se refiere a su antecesor:

Los alemanes son los únicos que emplean hoy la palabra estética para designar lo que otros denominan crítica del gusto. Tal empleo se basa en una equivocada esperanza concebida por el destacado crítico Baumgarten. Esta esperanza consistía en reducir la consideración crítica de lo bello a principios racionales y en elevar al rango de ciencia las reglas de dicha consideración crítica $(\S 1)$.

A partir de la afirmación de Kant, podemos establecer nuestra primera pregunta de investigación: ¿cuáles son los principios racionales que establece Baumgarten para fundar una ciencia del conocimiento sensible?

El trazado que debemos hacer para identificar el caso particular de Baumgarten, en el contexto del racionalismo del siglo XVII y XVIII, implica un gesto inverso al propuesto por Kant. Es decir, asumir que, precisamente, es necesario volver a las referencias que permitieron la constitución de su doctrina estética, a partir de la ampliación del foco de identificación del rango de influencias que pudo haber tenido Baumgarten en el contexto de la filosofía leibniziano-wolffiana, sin pasar al concepto de gusto que llegará justo a fines del siglo XVIII. De alguna manera, yendo más allá de la simple asociación reduccionista que hace Kant, debemos aceptar que Baumgarten no tuvo la intención de establecer una critica del gusto, sino una ciencia del conocimiento sensible, y detenernos a entender su alcance.

De este modo, si vamos al principal eje referencial en su obra, surge el racionalismo inmediatamente anterior a Baumgarten como fuente. Debemos situarnos al menos medio siglo antes, cuando la desaparición de la figura de Descartes marcaba el panorama filosófico. Luego, en vez de seguir la vía tradicional de influencia, nos fijamos en la recepción crítica del cartesianismo que asume Leibniz. Reparo que, a su modo, fue seguido y asumido como proyecto general de distanciamiento del cartesianismo por el propio Wolff en todo su sistema filosófico. Este filósofo, en su Metafísica Alemana (1720), menciona a Descartes desde el $\S 3$, refiriéndose al hecho de que el modelo de 
conocimiento del alma en relación con el cuerpo alude a las dificultades que habría tenido Descartes para distinguir claramente cada facultad del alma y sus modificaciones (35).

Entonces, una segunda pregunta para nuestra investigación sería: ¿en qué grado los principios racionales que busca establecer Baumgarten, siguiendo la crítica de Leibniz y Wolff a Descartes, se diferencian radicalmente de aquellos propuestos por Descartes mismo?

Bajo estas dos interrogantes es posible recorrer los escritos estéticos de Baumgarten e identificar un factor determinante para el análisis de su obra, el que resalta al seguir las variaciones que surgen de las coincidencias conceptuales, aunque no siempre terminológicas - como bien señala Pimpinella- en los textos de Baumgarten (Wolff e Baumgarten 15). Con este fin, primero, tomaremos un aspecto particular del léxico estético, de modo que nos permita vislumbrar la fuerza con que el racionalismo previo a Baumgarten aflora a partir de las fuentes bibliográficas en las que se funda la disciplina estética moderna.

Cuando vamos al texto mismo de Baumgarten, podemos apreciar cómo su búsqueda de una gnoseología de la experiencia está fuertemente marcada por un conocimiento erudito de las fuentes clásicas, tanto de la poesía como de la filosofía y la historia. Estas menciones están de tal modo presentes en sus textos que no podemos dejar de preguntarnos por un particular contraste, entre la propuesta clásico-erudita de Baumgarten y aquella que el modelo racional cartesiano incluía como parte central de su método científico, en una oposición radical hacia las fuentes clásicas de la filosofía y la cultura (Discours de la methode 1637). De este modo, sin constituirse en una pregunta de investigación en sí misma, en este breve estudio, es fundamental señalar y de alguna manera establecer este parámetro comparativo, porque es otra forma de describir la transición que representa la obra de Baumgarten en el contexto del siglo XVIII. Nuestro filósofo con su articulación referencial, entre Descartes Leibniz y Wolff, pero también de Horacio, Cicerón y Virgilio, trae al presente autores que desaparecen de entre los índices onomásticos de los tratados de Descartes. Esta operación referencial le permite a Baumgarten indagar en problemas filosóficos que integran áreas como la poética y la retórica, a una parte de la filosofía, específicamente a la metafísica.

El filósofo berlinés asume la tarea de establecer una ciencia del conocimiento sensible y conjuga en sus investigaciones una terminología de acuerdo al legado de sus antecesores, quienes a su vez buscaban perfeccionar el método racional y corregir sus falencias. Leibniz, en su texto de noviembre de 1684 titulado Meditaciones sobre el conocimiento, La Verdad y las Ideas, reconoce abiertamente el hecho de que a pesar de "las vivas controversias acerca de las ideas verdaderas y falsas" y dada la importancia del tema, "ni siquiera el propio Descartes lo ha tratado en forma satisfactoria en parte alguna" (314) y por esta razón él presenta "brevemente" algunas "precisiones".

¿En qué consisten las precisiones que hace Leibniz a Descartes y, por ende, cómo podemos iniciar el proceso de identificación de las corrientes de influencia que llegan hasta Baumgarten desde el racionalismo cartesiano? Es otra de las preguntas que surgen de la lectura de Baumgarten. Las precisiones que hace Leibniz a Descartes permiten identificar las oleadas críticas que alcanzan hasta nuestro filósofo. Un aspecto 
por el que podemos abordar lo descrito antes, es la coincidencia en la utilización, por parte de estos cuatro filósofos mencionados hasta ahora (Descartes, Leibniz, Wolff y Baumgarten), de parámetros lumínicos para referirse al modelo racional, a partir de nociones tradicionales de claridad, distinción, versus oscuridad y confusión. Descartes utiliza en muchos pasajes de sus obras, así como en su amplia correspondencia, conceptos como, por ejemplo, los propuestos en los Principia philosophice: "Llamo clara a aquella [idea] que está presente y manifiesta al espíritu atento [...] Distinta, en cambio, es aquella que, siendo clara de tal modo está separada y recortada de todas las otras, que no contiene en sí nada más que lo que es claro" (AT VIII 13 y 22).

Por su parte, Leibniz en las Meditaciones sobre el conocimiento [...] escribe: "En efecto, el conocimiento es oscuro o claro y el claro además confuso o distinto y el distinto es inadecuado o adecuado, y también simbólico o intuitivo; y si es simultáneamente adecuado e intuitivo es sumamente perfecto" (314). Ahora bien, tal como aclara Ferraris, la noción de un conocimiento simbólico, por parte de Leibniz, incorpora un ámbito que ya cruza lógica y poética. Tal como lo concretará Baumgarten a través de la incorporación de lo simbólico en tanto conocimiento discursivo (Estetica 44-45).

Wolff, en la Metafisica alemana, en el capítulo III de la primera parte, trata extensamente el modelo de la percepción, al que ya no tan solo agrega la complementación simbólico discursiva de Leibniz, sino además incluye directamente la asociación de pensamiento y lenguaje como elementos inseparables (\$195). Ahora bien, ¿por qué la confluencia de términos es un índice tan importante para un estudio como este? Primero, porque asume la complejidad que representa el modelo lumínico que aporta el propio Descartes al momento de describir la experiencia gnoseológica. Segundo, porque tal como señala Blumenberg en Paradigmas para una metaforología, no podemos aceptar el hecho de limitar conceptos como claro-distinto-oscuro y confuso al ámbito puramente cartesiano, ya que desde una perspectiva histórica es notorio que estos conceptos han sido utilizados en el contexto de la retórica clásica por figuras como Cicerón, Quintiliano y otros (49-59). Por lo tanto, el legado terminológico no se fundaría en un aspecto enfocado puramente en el racionalismo cartesiano por parte de Baumgarten, sino en un modelo mucho más amplio relacionado con la incorporación, gracias al novedoso esquema de categorización de las ideas en Leibniz, el que, como decíamos antes, incorpora a través del concepto "simbólico", no solo lo discursivo lingüístico, sino además una dimensión para lo sensible en sentido amplio, es decir, que conlleva una variación de la noción de experiencia (en Leibniz y Wolff) y, paralelamente, su ampliación a la noción de experiencia estética (en Baumgarten). Esto también aflora en el contexto cartesiano, por distintas razones, y a pesar de todos los esfuerzos de reintegración histórica de la terminología, es difícil dejar atrás los prejuicios de originalidad que rigen los principales modelos historiográficos de la filosofía, los que dificultan el flujo entre doctrinas que parecieran distantes pero que, ya sea por concordancia o discordancia-como es el caso aquí- son dependientes en términos históricos y también conceptuales.

Un ejemplo bien conocido de esta complejidad que acabamos de precisar pero que no siempre es considerado - como bien señala Blumenberg para el caso de Descartes- es el que corresponde a la gravitación de los principales textos de patrología, con figuras como Orígenes (siglo III) y Lactancio (siglo IV), donde estos mismos conceptos forman 
parte de una concepción de la experiencia, establecida por el principio de la verdad en cuanto luz (Paradigmas 49-59). Esto mucho antes que se asuma la relación gnoseológica de dicha terminología y la consecuente derivación de una disciplina estética.

En este mismo sentido, Benedetto Croce, en su Estetica, 1902, en el capítulo dedicado al cartesianismo, considera que los hechos que llamamos estéticos "se identificaban con la cognición confusa en Descartes, la que puede ser clara sin por eso ser distinta" (260). Esta terminología, para Croce, se deriva de la Escolástica, y es sugerida de un modo particular por la doctrina de Duns de Scoto, quien tuvo reediciones y experimentó cierto reestablecimiento en las discusiones doctorales en el siglo XVII.

De este modo, el filósofo italiano prueba su afirmación remitiéndonos a los capítulos de su libro sobre estética medieval, porque identifica el momento de las disputas entre nominalismo y realismo como una situación de quiebre, tan importante como la que instala Descartes en su momento, pero, referido al medioevo y a las relaciones entre verbo y carne, pensamiento y palabra. Sin embargo, es necesario, al mismo tiempo, intentar reconstruir el itinerario que ha seguido la discusión de los aspectos racionalistas del pensamiento de Baumgarten, lo que significa distinguir dos momentos. Primero, aquel que acompañó la recepción de su obra inmediatamente después de la publicación de la Estetica, y otro, muy diverso, en la discusión que se ha dado durante el siglo XIX y XX, al momento de establecerse los primeros tratados donde se da cuenta de una historia de la estética como disciplina autónoma, que reconoce su centro en la noción de juicio de gusto.

Esta perspectiva terminológica puede parecer extremadamente ambiciosa, al mismo tiempo que restringida, considerando un estudio como el que presentamos. Su relevancia a la hora de entender el giro que representa la unión de una metafórica lumínica de la verdad, a través de siglos y siglos, así como el peso que representa la imagen misma de la verdad como luz, a la hora de establecer su equivalencia como ciencia del conocimiento sensible, suma otro componente a la razón como sistema. Es paradójico, por lo menos, pensar que es esencial para entender que desde el contexto más antiguo hay una consciencia de este doble juego conceptual, el que implica que una idea sea clara u oscura en sí y que, al mismo tiempo, lo que define la idea son conceptos que implican su percepción sensible. Esta concepción de la experiencia de la idea, que es metafórica por cierto, muestra una preocupación por el aspecto estético del concepto, más que por su manifestación como idea pura.

En la confluencia generada por una metafórica lumínica en cuanto estética, ciencia del conocimiento sensible, veremos que si desplazamos estas imágenes, como percepción o apercepción sensible, pero, además asumimos el diagrama lumínico para la verdad, estaremos moviéndonos bajo un fascinante efecto retórico general. La posibilidad que nos ofrece Baumgarten implica un modelo epistemológico de la experiencia de la idea, en cuanto enunciación o "discurso sensible", tal como lo llama en sus Meditaciones acerca del poema $§ 1$. Con esta ampliación de un conocimiento que suma el aspecto sensible de la experiencia racional, Baumgarten logra incorporar la experiencia en un espacio o dimensión menos reñido con nociones de racionalidad pura, extrasensible. Ferraris va más allá y afirma que, la propuesta de Baumgarten, se 
trata directamente de una dimensión donde "la evidencia estética (claridad) y aquella lógica confluyen" (41). De manera que, considerando las modificaciones propuestas por Leibniz, es en Descartes donde se establecen los principios estéticos fundamentales (postaristotélicos) que asume la modernidad. En este sentido, debemos entender que al menos en el caso de Descartes, el racionalismo tiene una carga en menor medida neoplatónica, que es como la tradición filosófica ha tratado de enmarcarlo. Aunque, quizás, en el caso de Leibniz, este ambiente de confluencia estético-racional se funda sobre pilares más lingüísticos que la semiología pasional y racional cartesiana, que se queda en un ámbito más tradicional de la concepción de la experiencia, bien anterior a los aportes del empirismo inglés con los que Leibniz dialoga.

No obstante, Annie Becq, en su libro Genèse de l'esthétique française moderne (1680-1814), afirma que los parámetros del racionalismo sirven para pensar la experiencia y que otro muy diverso es el proceso de establecimiento de una la legislación sobre las reglas del arte que se da el siglo XVIII (18). Esta misma estudiosa nos anuncia una consideración importante a la hora de volver sobre el curso de aquella transición entre el siglo XVII y XVIII que vio nacer la estética, subrayando el cuidado que debemos tener en "la ilusión retrospectiva" (18). Efecto historiográfico consistente en suponer que términos, por ejemplo, como el de razón, puedan poseer hoy un contenido idéntico a aquel que le dieron los estudiosos del siglo XVII. Sin duda, en la medida en que busquemos seguir las distintas vertientes en relación con una posición filosófica definida, veremos la aparición de un segundo peligro en el proceso de identificación de las fuentes del racionalismo cartesiano en las primeras estéticas del XVIII, que consiste en la búsqueda de analogías y concordancias por relaciones de filiación. Como afirma Becq, la primera generación del siglo XVII, contemporánea a Descartes, está determinada por las circunstancias históricas y morales. El racionalismo, en los inicios del siglo XVII, surge para algunos estudiosos como la forma de una exigencia apasionada de estabilidad y de claridad de análisis. Nuestro estudio, en su medida, busca interpretar estos espacios y quiebres.

Dicho esto, vayamos brevemente a la noción de sensibilidad que proyecta el modelo de conocimiento sensible en Baumgarten, en relación con la noción de experiencia bajo la variable lumínica. Para algunos estudios se trata de una versión del autómata cartesiano, para otros, de la mónada con consciencia de Leibniz. Para incorporar aquellos dos aspectos principales, primero, la mente, casi como si se tratara de un órgano superior específico de la experiencia; y, luego-sometido-el cuerpo, del que Baumgarten despliega una sensibilidad nerviosa, discursiva, poética y racional. Es en estos ejes donde situa las distintas formas gnoseológicas que vinculan la estética con lo que él mismo llama "conocimiento sensible". En el primer parágrafo de su Astetica plantea la siguiente definición: "§1. La estética (teoría de las artes liberales, gnoseología inferior, arte del pensamiento bello, arte análogo de la razón), es la ciencia del conocimiento sensible (cognitionis sensitivae)".

La acepción que propone nuestro filósofo de un "concocimiento sensible" comprende la experiencia estética como una experiencia racional del cuerpo y sensible de la razón. Se trata de una percepción múltiple que no tan solo tiene un carácter de dato y traza (nota) para la sensibilidad y la razón, sino que plantea una figuración mental 
que llama fuerza representativa (vis repraesentativa), determinada por la sensibilidad y sus formas perceptivas y representativas. No solo considerando la recepción sensible, sino su recuperación figurativa, a través de los signos y señales propias de las ideas, la memoria y las emociones.

Esta disposición de la propuesta estética y poetológica de Baumgarten, centrada en el conocimiento sensible, plantea una pregunta concreta formulada con total exactitud por Frederick C. Beiser en su libro Diothimas Children: german aesthetic rationalism from Leibniz to Lessing: “¿Por qué las sensaciones son cognitivas? Es suficiente afirmar la teoría general de que todas las representaciones son cognitivas; necesitamos saber más específicamente por qué las sensaciones lo son" (139).

Una de las principales relaciones que establece el propio Baumgarten en su Astética es la identificación, como dijimos antes, de una fuerza representativa derivada de la experiencia (vis repraesentativa). Pero esta noción de experiencia no es simplemente una relación unívoca con la exterioridad, sino que implica -a partir de los parámetros leibniziano-wolffianos- un nuevo modelo de experiencia y de experiencia estética. Modelo que dista, sin duda, de aquel planteado por Descartes en tratados como El Mundo (póstumo) o el Tratado de las pasiones (1649). La pregunta de por qué las sensaciones son cognitivas está determinada por la noción de la experiencia que Leibniz ofrece y por la reelaboración que Wolff traduce en su Psychologia empirica (1732). Contexto referencial que nos permite adelantar la conclusión de que el trazado que establece Baumgarten tiene fuertes rasgos "monadológicos" heredados de sus antecesores, así como aquellos que siguen el modelo de los afectos del Tratado de las pasiones de Descartes.

De este modo, lo que se despliega a través de la confluencia estético-racional no es tan solo un cuerpo y una mente, sino además una noción de mundo proyectado como un universo (cosmos y heterocosmos) que la filosofía de Baumgarten concibe para unificar de manera amplia e integrada la experiencia en sí como fundamento. Beiser afirma:

Para Baumgarten, el universo es una unidad sistemática, una totalidad armónica, y cada evento sucede solamente en virtud del universo entero, actuando a través de él. De ahí que defina cualquier estado presente del universo, como la totalidad de su estado en sus partes simultaneas (Metafisica §369). Es precisamente este status mundi lo que es representado en una sensación. La sensación no es simplemente un evento dentro mío sino el universo entero tal como se manifiesta a través de mí. Por tanto, me muestra, en la frase leibniziana, el universo entero desde mi punto de vista" (141).

Vamos entonces al pasaje de La monadología donde Leibniz presenta el principio del que Beiser proyecta su deducción:

$\S 61 \mathrm{Y}$ por consiguiente todo cuerpo siente todo lo que pasa en el universo, de modo tal que el que viera todo podría leer en cada uno lo que ocurre en todas partes he incluso lo que ha ocurrido y ocurrirá, advirtiendo en el presente lo que está alejado tanto según los tiempos como según los lugares [...] Pero un alma 
puede leer en sí misma solo lo que está representado en ella distintamente, no podría desarrollar de modo instantáneo todos sus repliegues, porque se extienden hasta el infinito (Monadología).

Baumgarten asume la tarea de establecer una ciencia del conocimiento sensible, introduciendo y aprovechando la noción de analogon rationis, conjugando en sus investigaciones una terminología que perfecciona el método racional cartesiano -definitivamente ampliado por Leibniz y Wolff- y aspira a corregir sus falencias terminológicas y conceptuales.

Los diferentes ejes que asigna Baumgarten al conocimiento, en un sentido amplio, y cómo este puede dividirse en una función cognitiva sensible más compleja, dada por su integración gnoseológica, en tanto razón análoga, es radicalmente contraria a una forma de conocimiento puro. Si volvemos a La monadología, veremos que Leibniz describe ya no un autómata como lo proyectó Descartes en la Meditaciones metafísicas, sino un molino, ya que busca representar los efectos externos de la causalidad mecánica ampliada y multiplicada en la mónada, diseñando una entrada para que la maquinaria tome conciencia del modelo general, es decir, experimente. Leibniz escribe:

$\S 17$ [Por otra parte,] nos vemos obligados a confesar que la percepción y lo que depende de ella es inexplicable por razones mecánicas, es decir, mediante las figuras y los movimientos. Y si imaginamos una máquina cuya estructura haga pensar, sentir, tener percepción, se la puede concebir de mayor tamaño conservando las mismas proporciones, de manera que se pueda entrar en ella como en un molino. Concedido esto, al visitarla por dentro solo se hallarán piezas que se empujan unas a otras y jamás algo con qué explicar una percepción (Monadología).

Este juego conceptual de representación, metáfora, donde una idea clara en sí, u oscura, define conceptos que implican no solo su percepción sino su manifestación, más que poseer una característica racional, refleja cómo el propio concepto implica su comprensión sensible, más allá de parámetros veritables, sino simplemente posibles. Sin embargo, si lo pensamos bajo un prisma retórico, como bien acepta la misma prosa de Baumgarten, veremos que se trata de un modelo epistemológico de la experiencia de la idea en cuanto enunciación o "discurso sensible". Es decir, manifestación verbal que el propio Baumgarten conecta en sus Meditaciones filosóficas acerca de la Poesía (1735):

$\S 4 \mathrm{El}$ discurso de las representaciones sensibles será sensible. Así como ninguno de los filósofos descendió a tal grado de profundidad de modo que con el puro intelecto haya penetrado enteramente todas las cosas, nunca quedándose fijo en el conocimiento confuso de ciertas cuestiones, y además prácticamente ningún discurso es tan científico e intelectual que no se encuentre a lo largo de todo su entrelazamiento ni siquiera una idea sensible, así, el que se aplica al conocimiento distinto puede hallar estas o aquellas representaciones distintas en el discurso sensible; este permanece, sin embargo, sensible, como el anterior permanece abstracto e intelectual.

Así, el conocimiento sensible, a través del principio de manifestación discursiva y su decodificación recíproca y simultáneamente, sensible y racional, resulta un vestigio 
elocuente de la intención desatendida en un autor que muchas veces es nombrado solo como aquel que acuñó el concepto de estética. Nombre que se utiliza como si se tratara de un umbral que celebra antiguos triunfos, pero que, al mismo tiempo, sirve de defensa para resguardar las formas actuales de maniobra de ese autómata cartesiano o del molino de Leibniz, que creemos haber transformado a la vida plena. En este mismo sentido, recordemos otro pasaje de La monadología, donde Leibniz hace la diferencia entre el autómata artificial cartesiano y su llamado autómata natural, del que deducimos el modelo de sensibilidad y experiencia estética en Baumgarten. Leibniz escribe:

§64 Cada cuerpo orgánico de un ser viviente es, pues, una especie de máquina o de autómata natural, que supera infinitamente a todos los autómatas artificiales. Porque una máquina, construida según el arte humano, no es máquina en cada una de sus partes; por ejemplo, el diente de una rueda de latón tiene partes o fragmentos que ya no son algo artificial y ya no tienen nada que caracterice la máquina respecto del uso al que estaba destinada la rueda. Pero las máquinas de la naturaleza, es decir, los cuerpos vivientes, son máquinas incluso en sus menores partes hasta el infinito. Es lo que constituye la diferencia entre la naturaleza y el arte, es decir, entre el arte divino y el nuestro.

No podremos resolver la diferencia que instala el pensamiento de Baumgarten, si no aceptamos que lo que en realidad imagina Leibniz, y lo que en el fondo trata de cumplir la ciencia del conocimiento sensible, con la tarea posterior de no tan solo imaginar un cuerpo racional sino, precisamente, asumir la imposibilidad de crear un cuerpo puramente racional y una mente puramente sensible, se debe al modelo mismo y su eje lumínico en sí, como estética.

Es tan potente el principio visual en la dinámica de la percepción en las Meditaciones sobre el conocimiento [...] que Leibniz plantea la imagen de la ceguera como límite de la posibilidad de una forma de experiencia cognitiva concreta:

Por esta razón no se le puede explicar a un ciego qué es el rojo, ni hacerles conocer a los demás cosas de este tipo a menos de ponerlos en presencia misma de la cosa y hacer que la vean, huelan o gusten o induciéndolos a recordar ciertas percepciones semejantes que experimentaron en el pasado. [...] En forma análoga vemos que los pintores y otros artistas saben a la perfección cuando algo ha sido realizado correcta o defectuosamente, aunque a menudo les resulte imposible dar razón a su juicio, y responden a quien los interrogan que echan de menos "un cierto no sé qué" en aquello que critican (315).

No es posible determinar si la precisión de Leibniz es buscadamente estética, pero, sin duda, su recuperación por parte de Baumgarten, en su modelo general de ciencia del conocimiento sensible, sí lo es. Leibniz vuelve sobre el conocido ejemplo que pone Descartes en las Meditaciones metafísicas (1641), el que citamos aquí extensamente como una manera elocuente de reenviar también al problema terminológico que sitúa a Baumgarten en su tiempo. Leibniz escribe: 
Así al pensar el quiliógono o polígono de mil lados iguales no siempre reparo en la naturaleza del lado ni en la de igualdad, ni en la del millar (o sea, del cubo de diez) sino que empleo en mi espíritu esas palabras (cuyo sentido se presenta a la mente por lo menos de un modo oscuro e imperfecto) en lugar de las ideas que tengo de ellas, pues recuerdo poseer su significado, aunque por el momento juzgo que es innecesario explicarlo. Suelo llamar a este tipo de pensamiento ciego o también simbólico [discursivo]: se lo utiliza no solo en el álgebra sino en la aritmética, y casi en todo. [...] De todo lo cual resulta ya manifiesto que no percibimos las ideas de aquellas cosas que también conocemos distintamente, sino en la medida en que empleamos un pensamiento intuitivo. Y sucede sin duda que a menudo creemos infundadamente poseer en el espíritu las ideas de las cosas, al suponer infundadamente que ya hemos explicado algunos de los términos que utilizamos. Y no es verdad o por lo menos es ambiguo lo que algunos dicen, a saber: que no podemos hablar de algo, entendiendo lo que decimos, si no poseemos la respectiva idea. Pues cualquiera sea el modo como entendamos cada una de estas palabras en particular o recordemos haberlas entendido antes, muchas veces sucede que se nos oculta la contradicción que quizás encierra la noción compuesta, dado que a menudo nos contentamos con ese pensamiento ciego y no proseguimos suficientemente la descomposición de las nociones (Meditaciones sobre el conocimiento... 316-317).

De esta forma, el universo terminológico que usa Baumgarten en sus tratados estéticos se vuelve en sí mismo una categorización estética por partida doble. Es decir, a partir de las fuentes que motivaron aquella ciencia del conocimiento sensible hecho discurso (simbólico este también), asimismo como en un modelo estético de la manifestación de la Idea bajo parámetros perceptivos o discursivos, es decir, estéticos y retóricos.

Ahora bien, para finalizar, el hecho de que Baumgarten organice sus fundamentos estéticos a través de un programa típicamente lógico tiene sus razones. El filósofo estaría en busca de una forma de ordenar el eje del problema permanente que implican los procesos metodológicos racionales, es decir, no dejar fuera el modelo mismo de pensamiento a la hora de pensar el modelo y sus ejemplos. De esta manera, Baumgarten le asigna un rol a la experiencia que, a pesar de estar formulado bajo criterios lógicometafísicos y, por lo tanto, absolutos, implica una iniciativa radical en la fundación de la estética como disciplina filosófica. Confluencia que, más allá de los avatares que traerá su mutación hacia la filosofía del arte, por un lado, y, hacia la estética como juicio de gusto, por otro, sus reflexiones se vuelven centrales para los interregnos que se crearán en la filosofía contemporánea entre ontología, fenomenología, hermenéutica e iconología (Ferraris 1997).

Baumgarten, motivado por esa especial circunstancia en la que ve la posibilidad de impulsar un área relegada de la metafísica, concibe la estética -entendida como ciencia del conocimiento sensible, como decíamos antes- a partir de la metafísica de Wolff, quien incluía en su esquema general, en la sección dedicada a la "Filosofía dogmática", la "Filosofía de las artes", compuesta: primero por Tecnología, luego Filosofía de las Artes Liberales, con un subconjunto integrado por Gramática, Retórica y Poética; y, finalmente, la Filosofía de la Medicina (Metafisica alemana). 
En resumen, Baumgarten recoge la premisa de Wolff que ilumina toda su producción filosófica: todo es pensable. Añadiendo una revisión exhaustiva del modo en cómo pensar la experiencia y, al mismo tiempo, hace el esfuerzo por incorporar la producción poética (en un sentido general de producción simbólica), revelando cuánto es consciente de las operaciones metafóricas mismas y la articulación de formas alternativas de enunciación filosófica. Todo esto, a partir de la elaboración de una lógica distinta a aquella que funda la articulación del conocimiento científico, ligado, a su vez, a un universo lógico también. Ese universo paralelo, Baumgarten lo establece a partir de la aplicación del concepto leibniziano de una razón análoga (analogon rationis). Aquella que nos permite ese otro sentido del sentido, el sentido figurado, la resolución de lo oscuro y el disfrute de la claridad de lo confuso: lo poético.

A este aspecto analógico o metafórico general debemos sumar, además, la distancia que se irá acrecentando, cada vez más, con la posterior instalación definitiva de la perspectiva kantiana, que confina esta coyuntura a los antecedentes precríticos del juicio de gusto. Y aunque pueda parecer extremo el imaginar esta temprana disyunción entre estética y filosofía del arte, ambas posturas responden a vertientes del programa general que Wolff buscaba fundar filosóficamente, a partir de cada ámbito del saber y del hacer (Pimpinella 14). No obstante, es difícil sintetizar las fuentes que fundan el programa de Wolff, al mismo tiempo que no podemos desconocer su formación cartesiana, así como una madurez leibniziana incontestable (Pimpinella 15). Baumgarten habría absorbido y potenciado este legado filosófico racionalista, y, si vamos a sus principales obras, veremos que es más que una coincidencia terminológica, es un modelo gnoseológico complejo, tanto en su primer escrito, Meditationes philosophica de nonnullis ad poema pertinentibus (1735), en la Metaphysica (1739), así como en la Aesthetica (1750-1758). Un conocimiento sensible, que no tan solo determinará una nueva acepción de razón, sino, por sobre todo, grafica la incontestable necesidad de la sensibilidad para la razón.

Referencias bibliográficas

Baumgarten, A. (1988), Esthétique de A.G. Baumgarten (J.-Y. Pranchère, ed.). L'Herne.

(2000), L'estetica (S. Tedesco, ed., \& A. L. F. Caparrotta, Trans.). Palermo: Aesthetica.

(1999), Riflessioni sulla Poesia (S. T. P. Pimpinella, ed.). Palermo.

Becq, A. (1984), Genèse de l'esthétique française moderne, de la Raison classique à l'Imagination créatrice 1680-1814. Paris: Albin Michel.

Beiser, F. C. (2009), Diothimas Children: german aesthetic rationalism from Leibniz to Lessing.

Blumenberg, H. (2003), Paradigmas para una metaforología (J. P. Velaco, Trans.). Madrid: Trotta. 
Borowsky, L. E. (1993), Relato de la vida y el carácter de Immanuel Kant (A. G. Ruiz, trad.). Madrid: Tecnos.

Croce, B. (1990), Estetica come scienzia dell'espressione e linguistica generale. Adelphi.

Descartes, R. (1996), Ouvres de Descartes. Paris: Vrin.

Ferraris, M. (1997), Estetica razionale. Milano: Raffaello Cortina.

Kant, I. (2000), Crítica de la razón pura (P. Ribas, trad.) Madrid: Alfaguara.

Leibniz, G. W. (2003), Escritos filosóficos (E. d. Olaso, ed.). Madrid: Machado Libros.

Pimpinella, P. (2005), Wolff e Baumgarten: Studi di terminologica filosofica (Olschki, ed.). Firenze.

Wolff, C. (2003), Metafísica alemana (R. Ciafardone, ed.). Milano: Bompiani.

(2000), Pensamientos racionales acerca de Dios, el mundo y el alma del hombre, así como sobre todas las cosas en general (Metafisica Alemana). (A. G. Ruiz, ed.). Madrid: Akal. 